\title{
Erratum to: Surgeons don't know what they don't know about the safe use of energy in surgery
}

\author{
Liane S. Feldman · Pascal Fuchshuber • \\ Daniel B. Jones - Jessica Mischna - Steven D. Schwaitzberg • \\ the FUSE (Fundamental Use of Surgical Energy ${ }^{\mathrm{TM}}$ ) Task Force
}

Published online: 14 December 2012

(C) Springer Science+Business Media New York 2012

\section{Erratum to: Surg Endosc (2012) 26:2735-2739 \\ DOI 10.1007/s00464-012-2263-y}

The list of FUSE members should have been positioned on the first page of the article as a footnote instead of underneath Table 1. Also, Malcolm G. Munro MD was inadvertently omitted from this list.

The online version of the original article can be found under doi:10.1007/s00464-012-2263-y.

Members of the FUSE (Fundamental Use of Surgical EnergyTM) Task Force include: Daniel B. Jones MD (Chair), Liane S. Feldman MD (Co-Chair), Pascal Fuchshuber MD (Co-Chair), Sharon L. Bachman MD, L. Michael Brunt MD, James Choi MD, Suvranu De ScD, Brian J. Dunkin MD, Warren Grundfest MD, Charlotte Gugliemi RN, Jeffrey W. Hazey MD, Scott Helton MD, Daniel M. Herron MD, David Iannitti MD, Gretchen Purcell Jackson MD, Stephanie Jones MD, Jarrod Kaufman MD, Leena Khaitan MD, Dean J. Mikami MD, William S. Richardson MD, Thomas N. Robinson MD, Marc Rozner MD, Steven D. Schwaitzberg MD, Daniel J. Scott MD, Victoria J. Steelman PhD, Thadeus L. Trus MD, J. Esteban Varela MD, C. Randy Voyles MD, Eelco Wassenaar MD and Malcolm G. Munro MD.

\section{S. Feldman ( $\square)$}

Department of Surgery, Steinberg-Bernstein Centre for Minimally Invasive Surgery and Innovation, McGill University Health Centre, 1650 Cedar Avenue, Room E19-117, Montreal, QC H3G 1A4, Canada

e-mail: liane.feldman@mcgill.ca

P. Fuchshuber

The Permanente Medical Group, Kaiser Medical Center, Walnut Creek, CA, USA

D. B. Jones

Department of Surgery, Harvard Medical School, Boston, MA, USA
J. Mischna

Society of American Gastrointestinal and Endoscopic Surgeons, Los Angeles, CA, USA

S. D. Schwaitzberg

Department of Surgery, Cambridge Health Alliance, Cambridge, MA, USA 